\title{
A Wide Lock-Range Referenceless CDR with Automatic Frequency Acquisition
}

\author{
Seon-Kyoo Lee, Young-Sang Kim, Hong-June Park, and Jae-Yoon Sim \\ Department of Electronic and Electrical Engineering, Pohang University of Science and Technology (POSTECH), San 31, Hyojadong, \\ Pohang 790-784, Republic of Korea \\ Correspondence should be addressed to Seon-Kyoo Lee, leesk@postech.ac.kr
}

Received 26 April 2011; Accepted 16 June 2011

Academic Editor: Woogeun Rhee

Copyright ( 2011 Seon-Kyoo Lee et al. This is an open access article distributed under the Creative Commons Attribution License, which permits unrestricted use, distribution, and reproduction in any medium, provided the original work is properly cited.

A wide lock-range referenceless CDR circuit is proposed with an automatic tracking of data rate. For efficient frequency acquisition, a DLL-based loop is used with a simple phase/frequency detector to extract 1-bit period of input data stream. The CDR, implemented in a $65 \mathrm{~nm}$ CMOS, shows a lock range of $650 \mathrm{Mb} / \mathrm{s}$-to- $8 \mathrm{~Gb} / \mathrm{s}$ and BER of less than $10^{-12}$ at $8 \mathrm{~Gb} / \mathrm{s}$ with low power consumption.

\section{Introduction}

Performance of a digital system is determined by the data rate of interchip communication as well as on-chip operating speed. As the development in process technology has successfully driven ever-increasing on-chip operating frequency, the off-chip interface is becoming the bottleneck in further improvement of system performance. For high-speed chipto-chip communication, serial link protocol has been widely adopted in various computer-to-peripheral interfaces and has achieved data rates of over $10 \mathrm{~Gb} / \mathrm{s}$ using differential signaling through a well-defined optical channel [1]. The widespread use of serial links for multipurpose, however, still presents some challenges which must be overcome by circuit design.

For wide-range CDR, two kinds of circuit schemes have been researched. One is the multirate CDR circuit with multiple reference clocks [2] or single reference clock with a programmable divider [3] or without reference clock $[4,5]$. The other is the continuous-rate CDR circuit with fractional$\mathrm{N}$ divider [6] or without an external reference clock [7-10]. The latter CDR scheme detects a change in the bit rate of the incoming data and adaptively controls the internal widerange VCO to track the bit rate without harmonic-lock issue. To extract the data frequency directly from an input data stream, several techniques have been presented with compli- cated state-machine-based frequency detectors [7-9] or using limited run length of $8 \mathrm{~B} 10 \mathrm{~B}$ coding $[10,11]$. The previous frequency acquisition circuits, however, cause large power consumption and area overhead. Therefore an efficient frequency acquisition algorithm is required to reduce complexity and power consumption.

This paper presents a $650 \mathrm{Mb} / \mathrm{s}$ to $8 \mathrm{~Gb} / \mathrm{s}$ referenceless CDR with an automatic tracking of data rate [12]. With a novel DLL-based frequency acquisition, the proposed dualloop CDR shows the highest performance in lock-range, power consumption, and size compared with previously reported continuous-rate CDRs.

\section{Circuit Description}

Figure 1 shows the proposed CDR which consists of a DLLbased frequency acquisition loop and a PLL-based loop for the clock and data recovery. In the frequency loop, the voltage-controlled delay line (VCDL) is automatically biased so that the delay of VCDL, T, would be equal to one bit duration, Tb. This frequency loop performs a two-step acquisition procedure which is a coarse lock with the coarse delay tracking (CDT) followed by a fine lock with the fine delay tracking (FDT). When CDT ends, FDT loop is enabled with the phase loop. A loss of lock detector (LLD) is included in the frequency loop to monitor a change in the data rate 


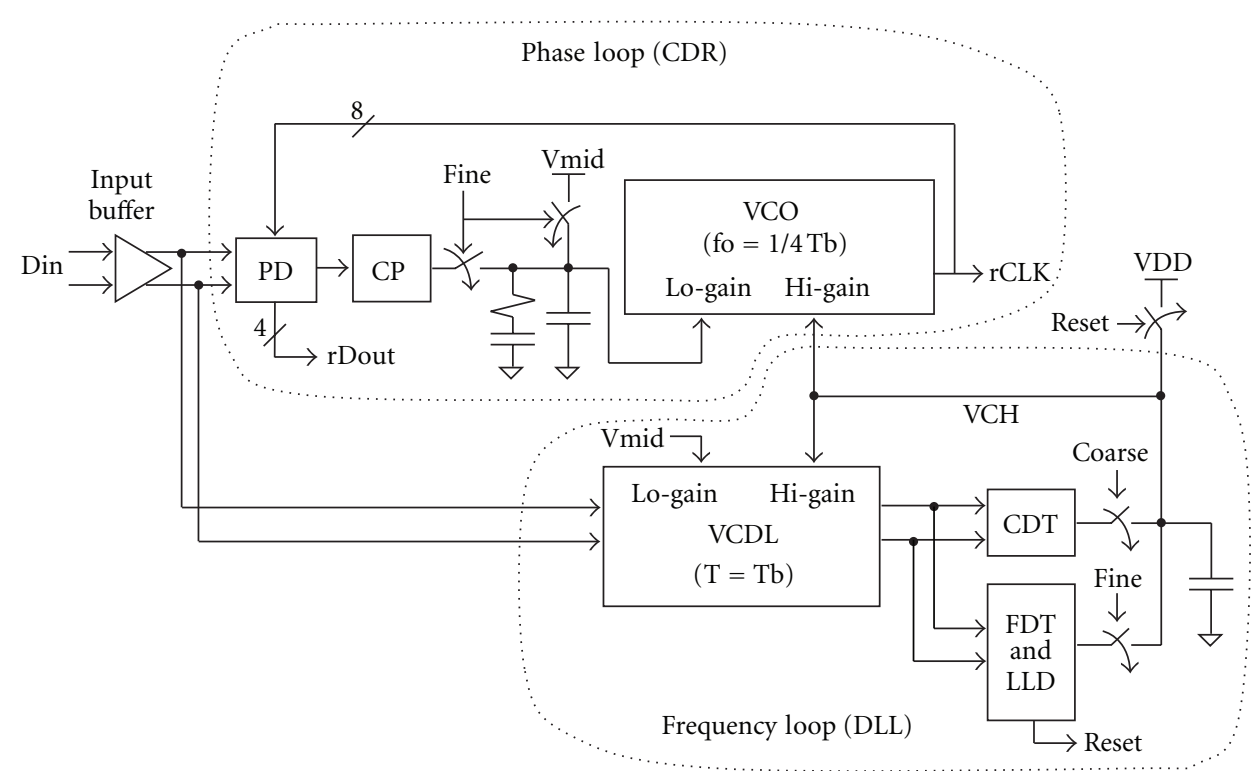

FIgURE 1: Architecture of proposed CDR.

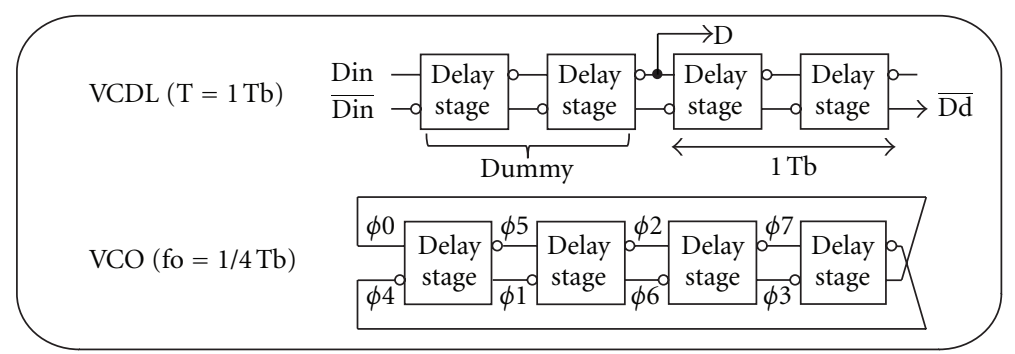

(a)

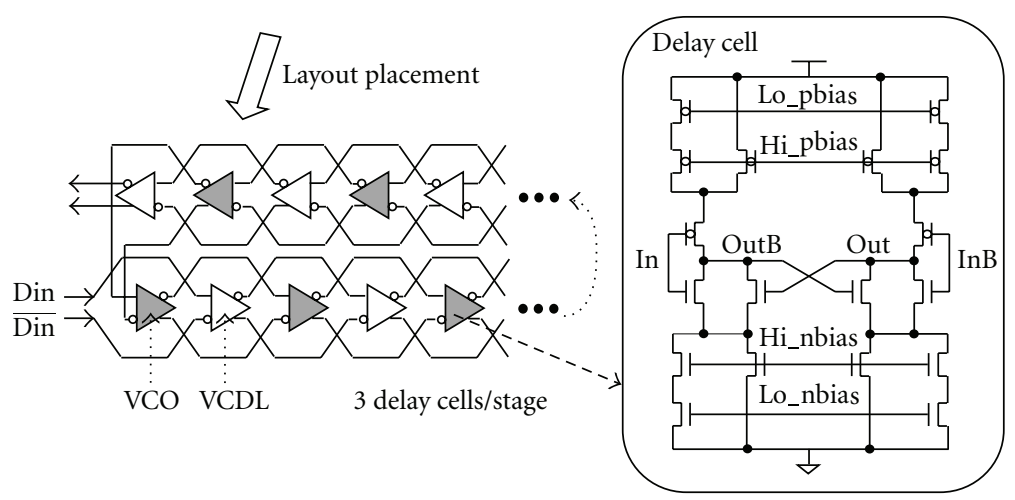

(b)

FIgURE 2: VCDL and VCO.

during the fine lock state. If LLD detects a change in the data rate, Reset signal is generated and it forces to restart from the coarse frequency lock again for automatic frequency acquisition. In the phase loop, a quarter-rate binary phase detector (PD) [13] is used with an 8-phase VCO.

Since matching between VCDL and VCO is an important assumption in the proposed frequency acquisition, identical delay circuit is used for both VCDL and VCO. The delay cell has hi-gain and lo-gain paths for the frequency lock and the phase lock, respectively.

2.1. VCO and VCDL. Figure 2 shows the circuit diagram of the delay cell. Hi_pbias and Lo_pbias are PMOS gate bias voltages generated by current-mirrored transformations from Hi_nbias and Lo_nbias, respectively. With the control range of from $0.5 \mathrm{~V}$ to $1.1 \mathrm{~V}$, the gain of the hi-gain path is 


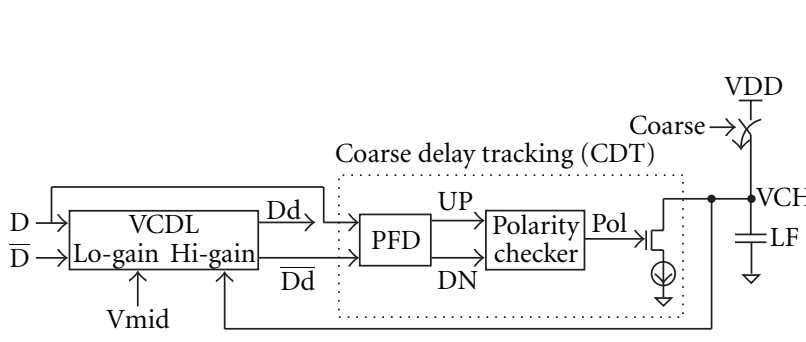

(a)

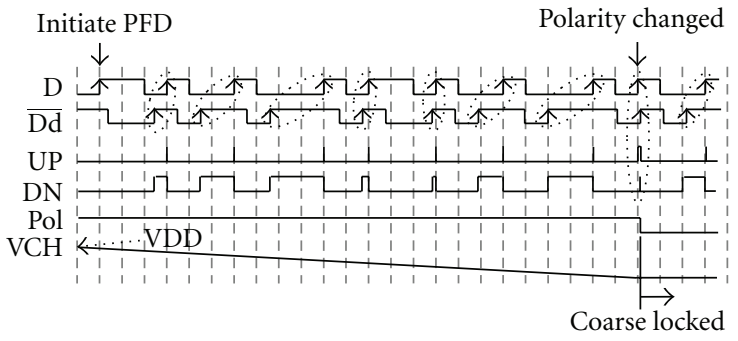

(b)

FIgURE 3: Coarse delay tracking.

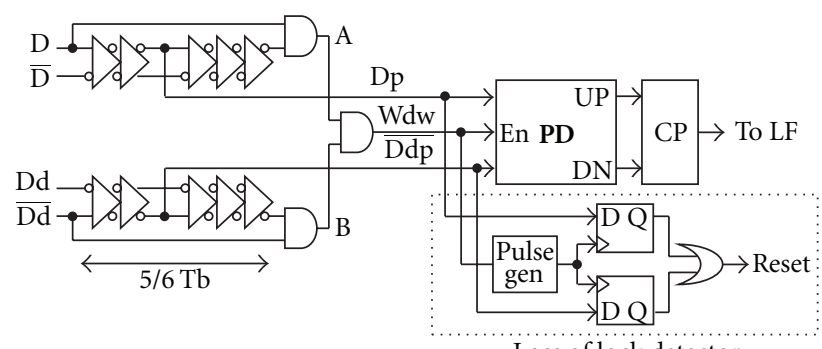

(a)

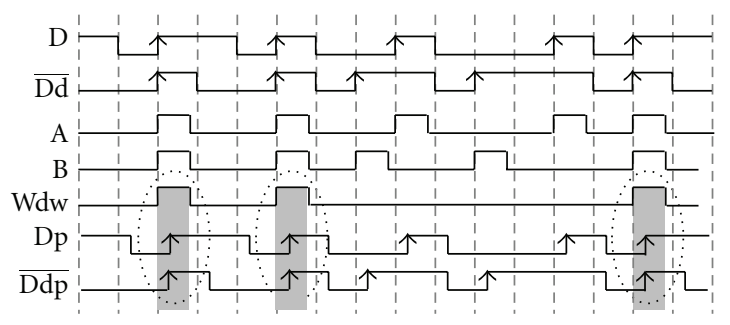

Fine locked state (data rate $\cong 4$ fo $)$

(b)

Figure 4: Fine delay tracking.

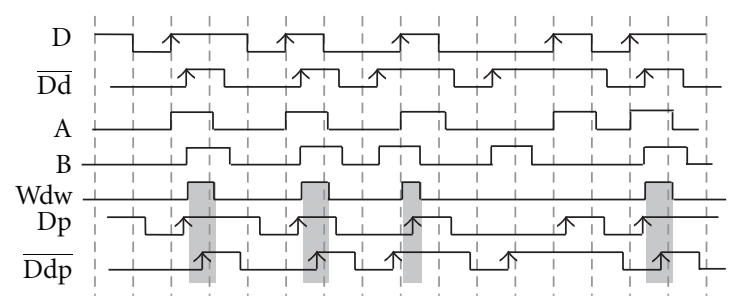

Data rate $>4$ fo

(a)

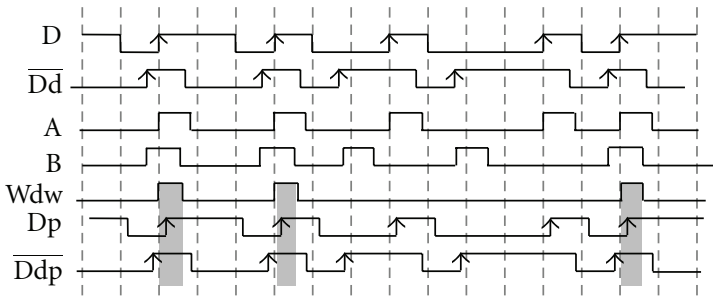

Data rate $<4$ fo

(b)

FIgUre 5: Cases of loss of lock.

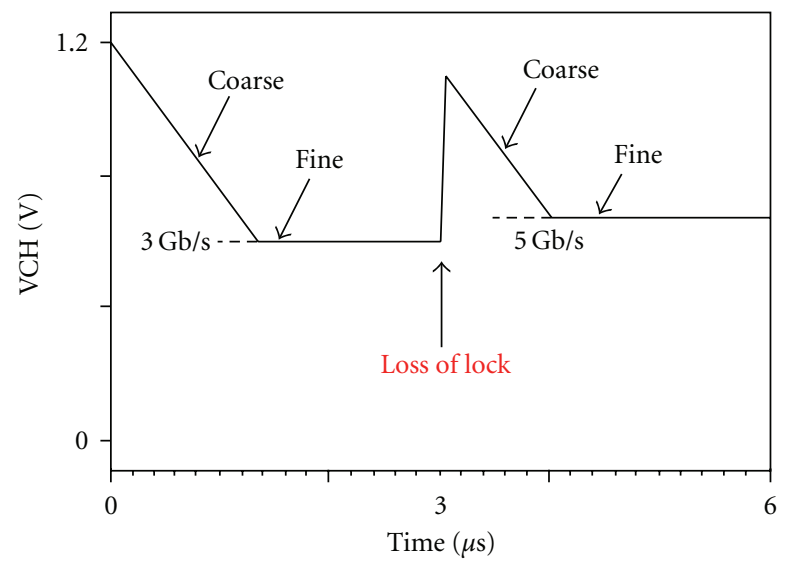

Data rate: $3 \mathrm{~Gb} / \mathrm{s} \longrightarrow 5 \mathrm{~Gb} / \mathrm{s}$ at $3 \mu \mathrm{s}$

(a)

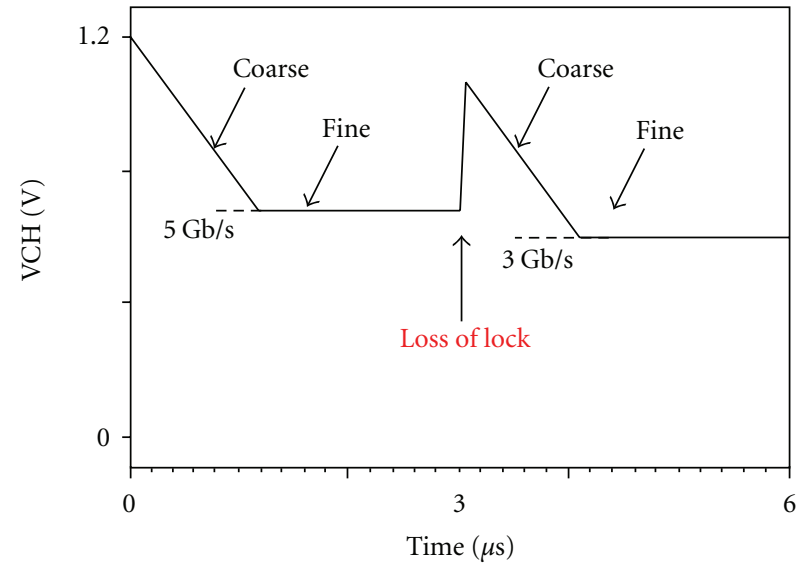

Data rate: $5 \mathrm{~Gb} / \mathrm{s} \longrightarrow 3 \mathrm{~Gb} / \mathrm{s}$ at $3 \mu \mathrm{s}$

(b)

FIGURE 6: Simulated automatic frequency acquisition in case of data rate changes. 


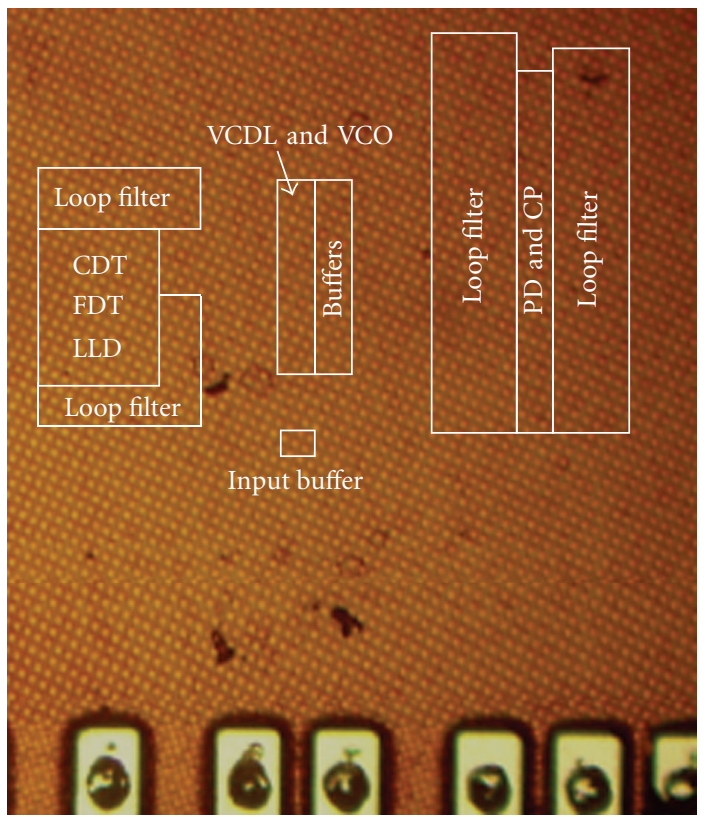

(a)

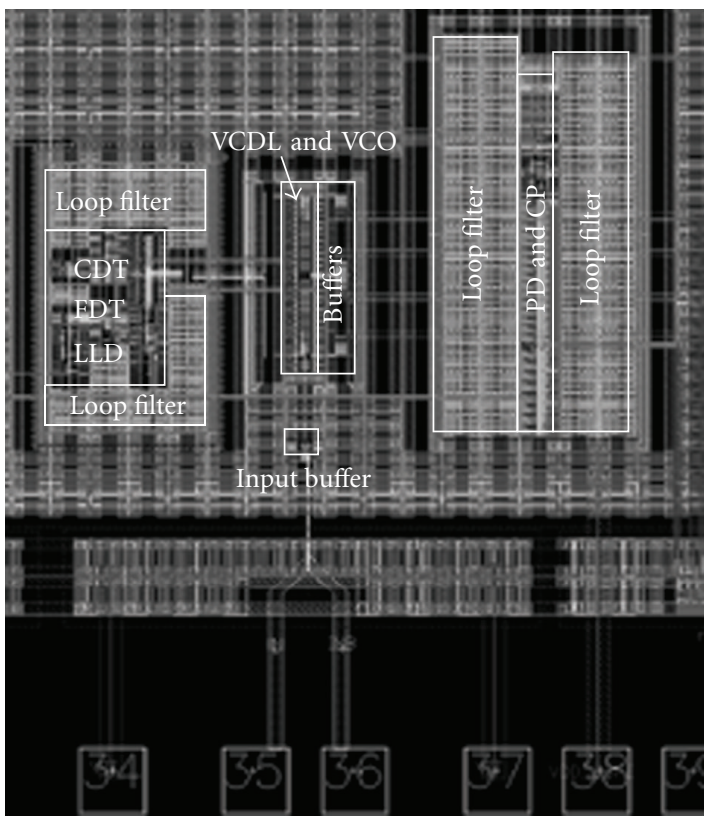

(b)

FIGURE 7: Photomicrograph and layout of the test chip.

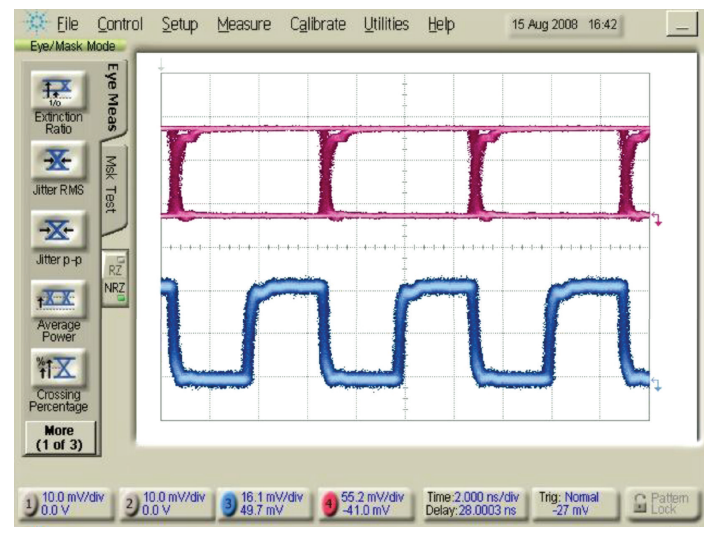

(a)

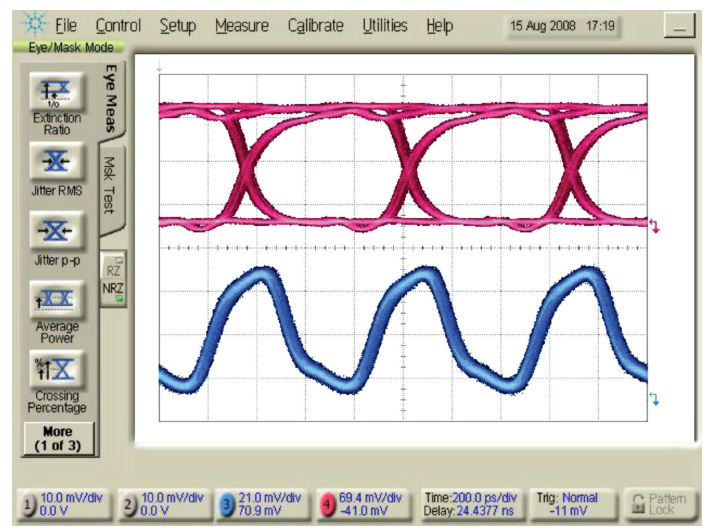

(c)

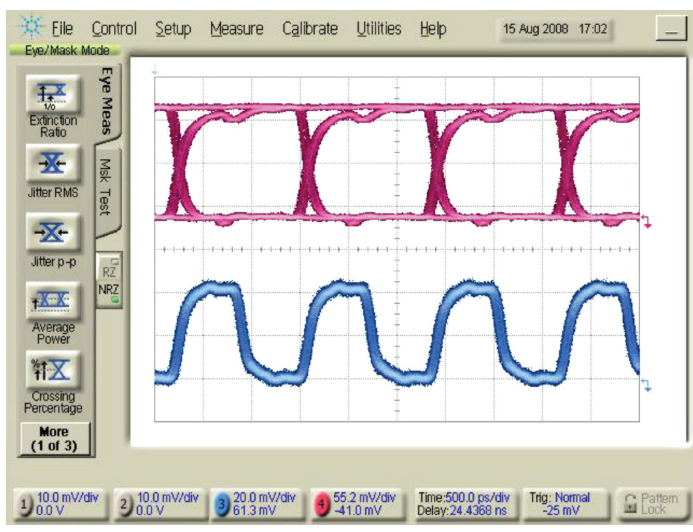

(b)

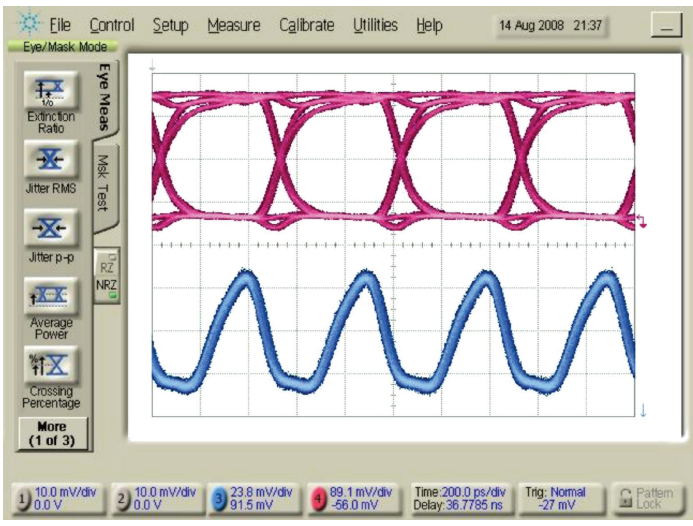

(d)

Figure 8: Measured eye diagrams and clock @650 Mb/s (a), $3 \mathrm{~Gb} / \mathrm{s}$ (b), $6 \mathrm{~Gb} / \mathrm{s}$ (c) and $8 \mathrm{~Gb} / \mathrm{s}$ (d). 


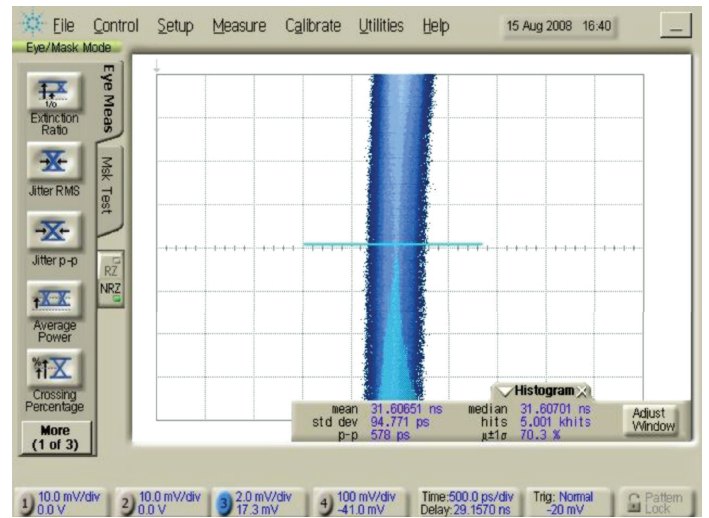

(a)

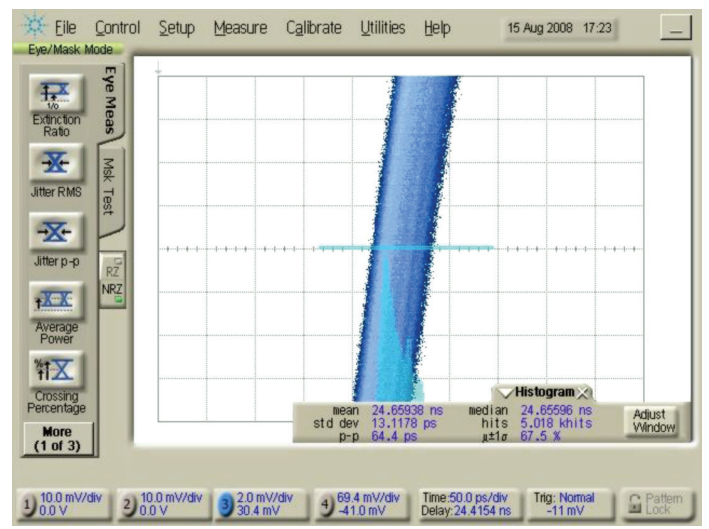

(c)

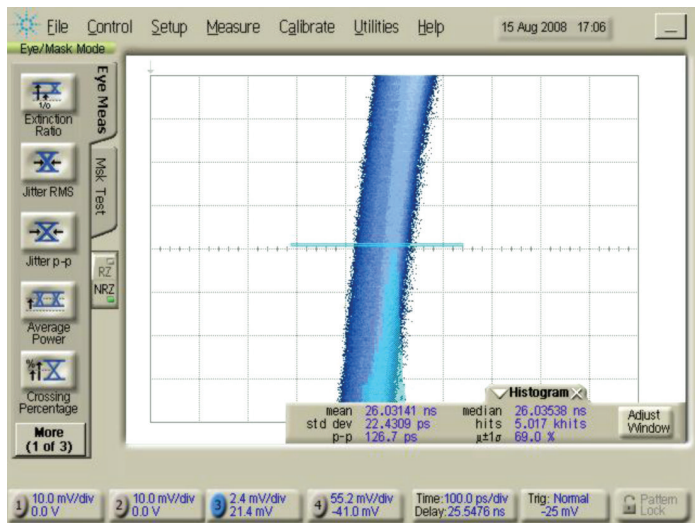

(b)

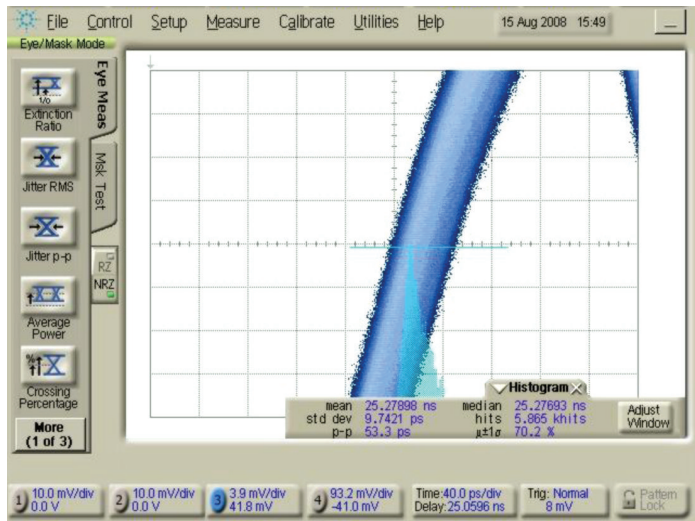

(d)

Figure 9: Measured clock jitter @650 Mb/s (a), $3 \mathrm{~Gb} / \mathrm{s}$ (b), $6 \mathrm{~Gb} / \mathrm{s}(\mathrm{c})$ and $8 \mathrm{~Gb} / \mathrm{s}$ (d).

designed to be $3.5 \mathrm{GHz} / \mathrm{V}$ for wide lock-range while the logain path is $150 \mathrm{MHz} / \mathrm{V}$ for better jitter performance. One delay stage is implemented by the cascade of three delay cells. But in actual layout placement, the total 24 delay cells for VCO and VCDL are alternated for improved matching.

2.2. Coarse Frequency Tracking Loop. Figure 3 illustrates the operation of CDT and how the delay of VCDL can be set to $\mathrm{Tb}$, which is performed by successful phase detection from a random NRZ bit stream. Before the coarse lock operation, the loop filter (LF) is initially charged to VDD so that VCDL would experience the minimum delay. When the coarse lock is started, the first coming rising edge of the input data $\mathrm{D}$ initiates the phase detection between the rising edges of $D$ and the inverse of the delayed input, $\overline{\mathrm{Dd}}$. The phase detection can be performed by a typical phase/frequency detector (PFD). Since the PFD is a sequential logic based on flip-flops, the initial value of PFD determines the pulse width of up/dn signal. So, the initialization by the first coming rising edge of $\mathrm{D}$ makes the desired initial value for the coarse operation. Since the initial delay of VCDL is set to be the minimum, PFD generates more DN pulse in the beginning. Then a pulldown current source decreases $\mathrm{VCH}$ until the polarity of PFD output changes to UP. Once the UP pulse becomes greater than DN pulse, the output of the polarity checker, Pol, is latched to low. It stops discharging $V C H$, which is the end of the coarse lock. This coarse delay tracking is performed through a hi-gain path, while lo-gain bias is fixed to the center of the control voltage range.

2.3. Fine Frequency and Phase Tracking Loop. After the coarse lock, FDT takes over the frequency loop. As shown in Figure 4 , the rising edges of $\mathrm{D}$ and $\overline{\mathrm{Dd}}$ generate autopulses on $A$ and $B$ with a pulse width of $5 / 6 \mathrm{~Tb}$ which is implemented by five-stage replica delay cells. An AND gate is used to generate a window signal, Wdw, to select appropriate rising edges for the phase detection. Dp and $\overline{\mathrm{Dpd}}$ are delayed signals of $\mathrm{D}$ and $\overline{\mathrm{Dd}}$, respectively, to make sure the rising edges be in the middle of Wdw when locked. With the replica delay cells, it is guaranteed that the rising edges of $\mathrm{Dp}$ and $\overline{\mathrm{Dpd}}$ are placed in the middle of Wdw pulse regardless of the change in bit rate. By accepting the rising edges of Dp and $\overline{\mathrm{Dpd}}$ only when Wdw is high, valid phase detection is achieved with a simple binary phase detector, and the output of PD drives a charge pump to perform the fine lock. Wdw, Dp, and $\overline{\mathrm{Dpd}}$ are also applied to LLD to detect a change of bit rate during the fine lock state. If LLD detects a change, the coarse lock procedure starts again for automatic tracking. If both rising edges of Dp and $\overline{\mathrm{Dpd}}$ are not in the Wdw pulse, it represents the loss of lock and reset signal is generated. Figure 5 shows the two cases of the loss of lock condition. 


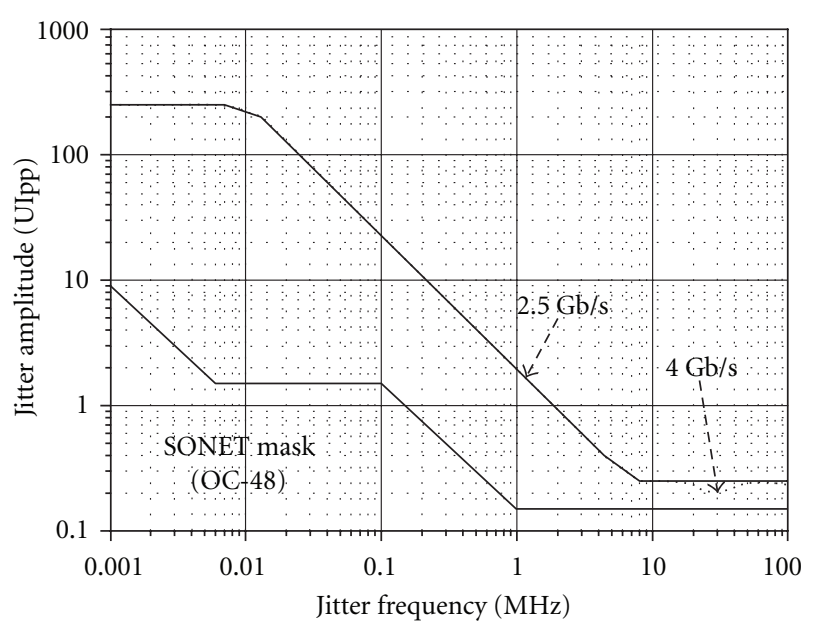

Figure 10: Measured jitter tolerance.

Figure 6 shows the simulated VCH when loss of lock occurs. When the input data rate is changed, the frequency loop detects it and automatically tracks the new data rate by starting the frequency acquisition again.

\section{Mesurements}

For verification, the proposed CDR circuit was implemented with a $65 \mathrm{~nm}$ CMOS technology as shown in the Figure 7. Active area was $0.108 \mathrm{~mm}^{2}$ including LF capacitors of $80 \mathrm{pF}$ in frequency tracking loop and $200 \mathrm{pF}$ in phase tracking loop. With a BER of less than $10^{-12}$, CDR operates at a lock range from $650 \mathrm{Mb} / \mathrm{s}$ to $8 \mathrm{~Gb} / \mathrm{s}$. Figure 8 shows measured eye diagrams of the quarter-rate recovered data and clock at different data rate.

Figure 9 shows measured quarter-rate recovered clock jitter. It was measured to $9.7 \mathrm{ps}_{\mathrm{rms}}$ and $53.3 \mathrm{ps}_{\mathrm{p}-\mathrm{p}}$ at the data rate of $8 \mathrm{~Gb} / \mathrm{s}$. The measured jitter can be decomposed into a pattern-dependent deterministic jitter of $20 \mathrm{ps}_{\mathrm{p}-\mathrm{p}}$ and a random jitter of $2.8 \mathrm{ps}_{\mathrm{rms}}$, respectively. As shown in Figure 10, the CDR also passed the OC-48 jitter tolerance specification at $2.5 \mathrm{~Gb} / \mathrm{s}$.

The CDR consumes power of $20.6 \mathrm{~mW}$ and $88.6 \mathrm{~mW}$ at $650 \mathrm{Mb} / \mathrm{s}$ and $8 \mathrm{~Gb} / \mathrm{s}$, respectively. Table 1 summarizes the performance of designed CDR. The proposed DLL-based frequency acquisition scheme achieved an efficient circuit implementation and shows suitability for the low-power and wide lock-range referenceless CDR.

\section{Conclusion}

A wide lock-range of $650 \mathrm{Mb} / \mathrm{s}$-to- $8 \mathrm{~Gb} / \mathrm{s}$ referenceless CDR circuit is proposed with an automatic tracking of data rate. For an efficient frequency acquisition in case of continuous data rate changes, a DLL-based loop is used with a simple phase/frequency detector. The CDR, implemented in a $65 \mathrm{~nm}$ CMOS, shows a BER of less than $10^{-12}$ with the best performance in lock-range, power consumption. The proposed DLL-based frequency acquisition scheme achieved
TABle 1: Performance summary.

\begin{tabular}{lc}
\hline Performance metric & Value \\
\hline Technology & $65 \mathrm{~nm} \mathrm{1P6M} \mathrm{CMOS}$ \\
Supply voltage & $1.2 \mathrm{~V}$ \\
Active area & $0.108 \mathrm{~mm}^{2}$ (including LF) \\
Data rate & $650 \mathrm{Mb} / \mathrm{s}-8 \mathrm{~Gb} / \mathrm{s}$ \\
& $88.6 \mathrm{~mW} @ 8 \mathrm{~Gb} / \mathrm{s}$ \\
Power consumption & $20.6 \mathrm{~mW} @ 650 \mathrm{Mb} / \mathrm{s}$ \\
& $9.7 \mathrm{ps} @ 8 \mathrm{~Gb} / \mathrm{s}$ \\
Jitter (rms) & $94.7 \mathrm{ps} @ 650 \mathrm{Mb} / \mathrm{s}$ \\
& $53.3 \mathrm{ps} @ 8 \mathrm{~Gb} / \mathrm{s}$ \\
Jitter (p-p) & $578 \mathrm{ps} @ 650 \mathrm{Mb} / \mathrm{s}$ \\
\hline
\end{tabular}

a simplified circuit realization and shows suitability for the low-power and wide lock-range referenceless CDR.

\section{Acknowledgment}

This paper was supported by Mid-Career Researcher Program through NRF Grant funded by the MEST (20110010685) and BK21 program.

\section{References}

[1] B. Razavi, "Design of high-speed circuits for optical communication systems," in Proceedings of the IEEE Custom Integrated Circuits Conference, pp. 315-322, San Diego, Calif, USA, May 2001.

[2] J. C. Scheytt, G. Hanke, and U. Langmann, "A 0.155, 0.622 and $2.488-\mathrm{Gb} / \mathrm{s}$ automatic bit-rate selecting clock and data recovery IC for bit-rate transparent SDH systems," IEEE Journal of Solid-State Circuits, vol. 34, no. 12, pp. 1935-1943, 1999.

[3] D. Belot, L. Dugoujon, and S. Dedieu, "A 3.3-V power adaptive 1244/622/155 Mbit/s transceiver for ATM, SONET/SDH," IEEE Journal of Solid-State Circuits, vol. 33, no. 7, pp. 10471058, 1998.

[4] M. H. Perrott, Y. Huang, R. T. Baird et al., "A 2.5-Gb/s multirate $0.25-\mu \mathrm{m}$ CMOS clock and data recovery circuit utilizing a hybrid analog/digital loop filter and all-digital referenceless frequency acquisition," IEEE Journal of Solid-State Circuits, vol. 41, no. 12, pp. 2930-2942, 2006.

[5] H. Nosaka, E. Sano, K. Ishii et al., "A 39-to-45-Gbit/s multidata-rate clock and data recovery circuit with a robust lock detector," IEEE Journal of Solid-State Circuits, vol. 39, no. 8, pp. 1361-1365, 2004.

[6] J. P. Frambach, R. Heijna, and R. Krösschell, "Single reference continuous rate clock and data recovery from 30Mbit/s to $3.2 \mathrm{Gbit} / \mathrm{s}$," in Proceedings of the IEEE Custom Integrated Circuits Conference, pp. 375-378, Scottsdale, Ariz, USA, May 2002.

[7] R.-J. Yang et al., "A 200-Mbps 2-Gbps continuous-rate clockand-data-recovery circuit," IEEE Transactions on Circuits and Systems I, vol. 53, no. 4, pp. 842-847, 2006.

[8] R.-J. Yang, K. H. Chao, S. C. Hwu, C. K. Liang, and S. I. Liu, "A 155.52 Mbps-3.125 gbps continuous-rate clock and data recovery circuit," IEEE Journal of Solid-State Circuits, vol. 41, no. 6, Article ID 1637602, pp. 1380-1390, 2006. 
[9] D. Dalton, K. Chai, E. Evans et al., "A 12.5-Mb/s to 2.7-Gb/s continuous-rate CDR with automatic frequency acquisition and data-rate readback," IEEE Journal of Solid-State Circuits, vol. 40, no. 12, pp. 2713-2724, 2005.

[10] M. S. Hwang, S. Y. Lee, J. K. Kim, S. Kim, and D. K. Jeong, "A $180-\mathrm{Mb} / \mathrm{s}$ to $3.2-\mathrm{Gb} / \mathrm{s}$, continuous-rate, fast-locking CDR without using external reference clock," in Proceedings of the IEEE Asian Solid-State Circuits Conference, pp. 144-147, Jeju, korea, November 2007.

[11] A. X. Widmer and P. A. Franaszek, "A DC-balanced, partitioned-block, 8B/10B transmission code," IBM Journal of Research and Development, vol. 27, no. 5, pp. 440-451, 1983.

[12] S.-K. Lee, Y. S. Kim, H. Ha, Y. Seo, H. J. Park, and J. Y. $\mathrm{Sim}$, "A $650 \mathrm{Mb} / \mathrm{s}-$ to-8Gb/s referenceless CDR circuit with automatic acquisition of data rate," in Proceedings of the IEEE International Solid-State Circuits Conference ISSCC 2009, vol. 52, pp. 184-185, San Francisco, Calif, USA, February 2009.

[13] J. D. H. Alexander, "Clock recovery from random binary signals," Electronics Letters, vol. 11, no. 22, pp. 541-542, 1975. 

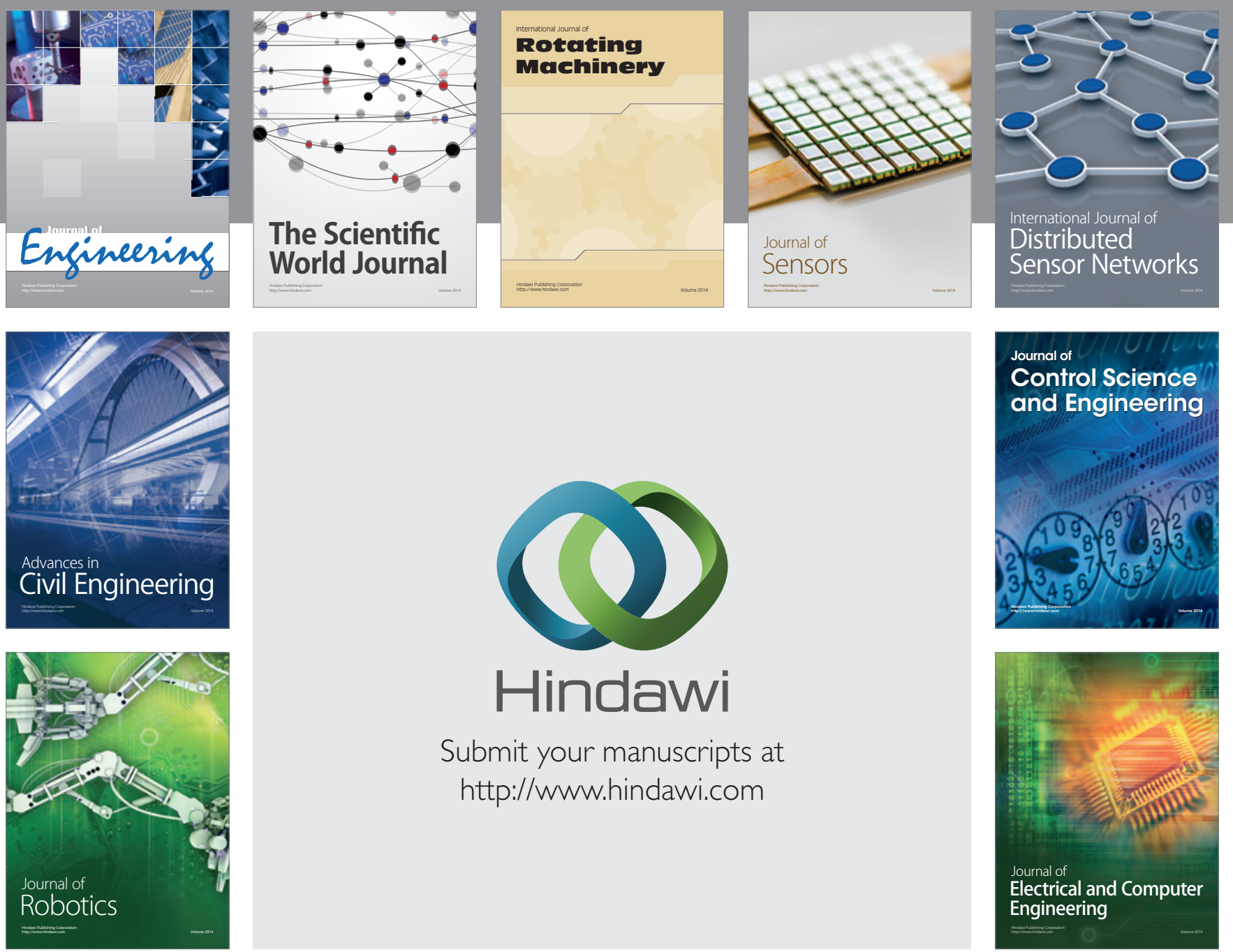

Submit your manuscripts at

http://www.hindawi.com
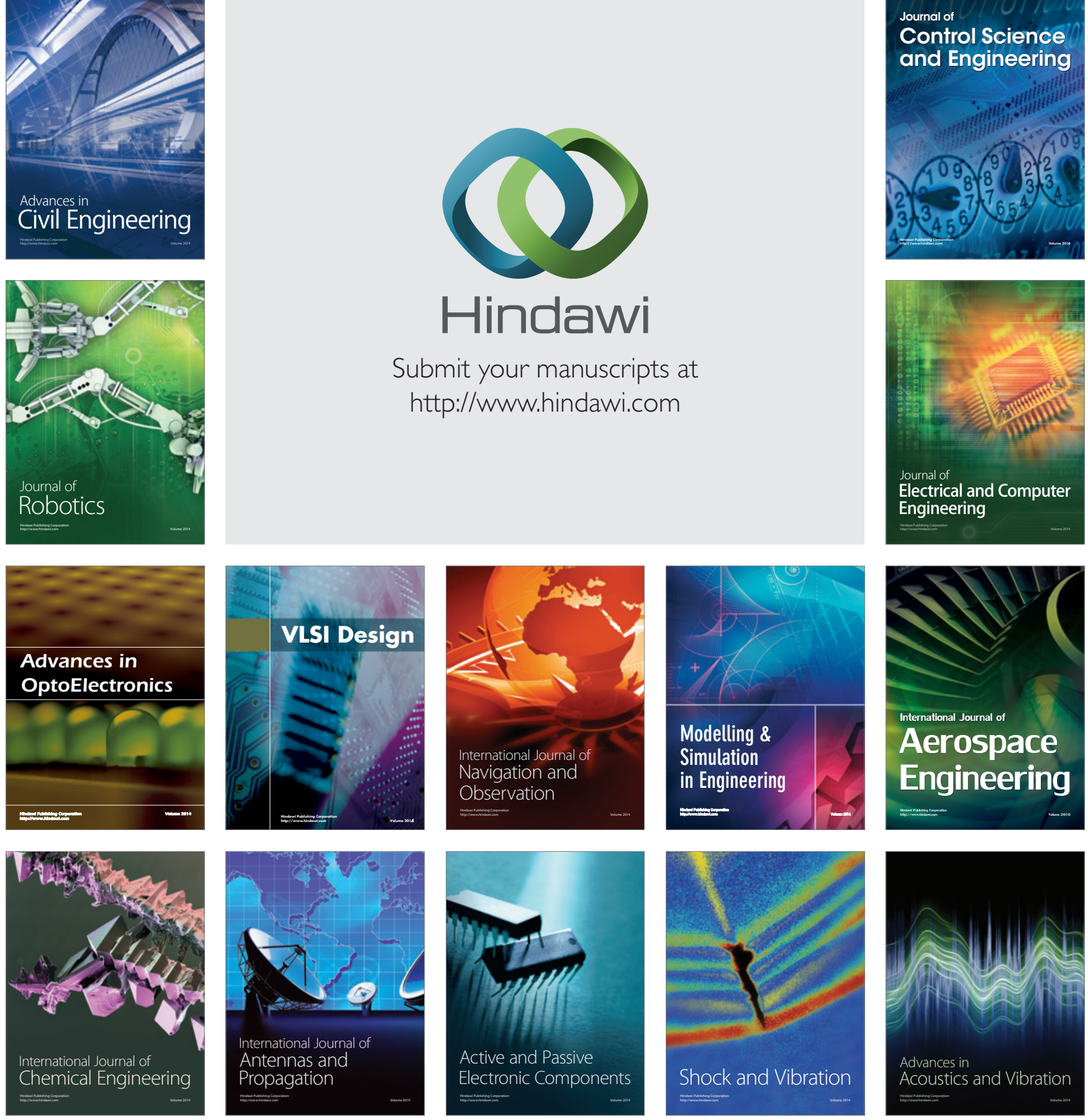